\title{
Are HoLEP Surgical Videos on YouTube Biased and Misleading or Are They Leading the Industry?
}

\author{
Omer Koras ${ }^{1}$, Fatih Gokalp², Ekrem Yıldırak ${ }^{3}$, Hakan Sigva ${ }^{3}$, Nezih Tamgaç ${ }^{3}$, Sefa Burak \\ Porgali $^{3}$, Bilal Kulak ${ }^{3}$, Ferhat Uçurmak ${ }^{3}$, and Sadik Gorur ${ }^{2}$ \\ ${ }^{1}$ Mustafa Kemal University Faculty of Medicine \\ ${ }^{2}$ Hatay Mustafa Kemal University \\ ${ }^{3}$ Affiliation not available
}

April 13, 2021

\begin{abstract}
Objective: In this study, we aim to evaluate the content and quality of the most relevant YouTube videos related to Holmium laser enucleation of the prostate (HoLEP) surgery. Materials and Methods: The keywords 'HOLEP', 'laser enucleation' and 'prostate enucleation' were used to perform a search on YouTube. Non-English language videos, videos with less than 4-minute duration, and repetitive videos were excluded. The reactions of the viewers to the videos were evaluated by recording the 'total views', 'views/month' and 'likes and dislikes' parameters. The data were divided into two groups based on the source of upload: Group 1 consisted of healthcare providers and Groups 2 comprised commercial companies and for-profit organizations. Results: A total of 117 videos were included in the study. A significant portion of the videos $(77.7 \%)$ had been uploaded by healthcare providers. There was no statistically significant difference between the uploading groups in terms of the DISCERN and GQS scores $(\mathrm{p}=0.484$ and $\mathrm{p}=0.108$, respectively). However, the PEMAT understandability and actionability scores were statistically significantly higher in Group $2(\mathrm{p}=0.004$ and $\mathrm{p}=0.022$, respectively). In addition, when the misinformation scale was evaluated, there were significantly more videos with high-degree misinformation in Group 2 (5.5\% vs 33.3, p=0.001). Conclusion: On video sharing platforms, such as YouTube, the number of reliable videos with accurate and appropriate guidance about diseases and treatments should be increased, and these videos should be allowed to be posted after they have been approved by relevant institutions, including healthcare associations and universities.
\end{abstract}

Are Holmium Laser Enucleation of the Prostate Surgical Videos on YouTube Biased and Misleading or Are They Leading the Industry?

\section{Abstract}

Objective: In this study, we aim to evaluate the content and quality of the most relevant YouTube videos related to Holmium laser enucleation of the prostate (HoLEP) surgery.

Materials and Methods: The keywords 'HOLEP', 'laser enucleation' and 'prostate enucleation' were used to perform a search on YouTube. Non-English language videos, videos with less than 4-minute duration, and repetitive videos were excluded. The reactions of the viewers to the videos were evaluated by recording the 'total views', 'views/month' and 'likes and dislikes' parameters. The data were divided into two groups based on the source of upload: Group 1 consisted of healthcare providers and Groups 2 comprised commercial companies and for-profit organizations.

Results: A total of 117 videos were included in the study. A significant portion of the videos (77.7\%) had been uploaded by healthcare providers. There was no statistically significant difference between the uploading groups in terms of the DISCERN and GQS scores ( $p=0.484$ and $p=0.108$, respectively). However, 
the PEMAT understandability and actionability scores were statistically significantly higher in Group $2(p$ $=0.004$ and $p=0.022$, respectively). In addition, when the misinformation scale was evaluated, there were significantly more videos with high-degree misinformation in Group $2(5.5 \%$ vs $33.3, p=0.001)$.

Conclusion: On video sharing platforms, such as YouTube, the number of reliable videos with accurate and appropriate guidance about diseases and treatments should be increased, and these videos should be allowed to be posted after they have been approved by relevant institutions, including healthcare associations and universities.

Keywords: HoLEP, patient information, social media, YouTube

\section{What's already known about this topic?}

Social media and video archive applications naturally consists of a large number of video contents that examine each subject or topic from different perspectives. However, available evidence has shown that patients can be exposed to low-quality, biased, and/or commercial videos, which can lead to dangerous consequences. Literature showed that huge proportion of videos containing medical information contained misinformation.

\section{What does this article add?}

Benign prostatic hyperplasia (BPH) incidence increases with age, reaching 80-90\% among the population. HoLEP that has gained popularity as a frequently preferred surgical method for BPH. Our study showed that the understandability and actionability scores of the videos uploaded by industry were statistically significantly higher compared to videos uploaded by non-profit healthcare. The number of videos with high-degree misinformation was significantly higher in uploaded by industry.

Acknowledgements: None

Funding: None

Conflict of interest: The authors declare that they have no conflict of interest.

Word count of text: 3000

\section{Word count of Abstract: 244}

\section{Introduction}

Benign prostatic hyperplasia (BPH) begins to be seen after the age of 40 years, and its incidence increases with age, reaching $80-90 \%$ among the population aged 70 to 80 years (1). Surgical treatment is applied in symptomatic BPH cases that do not benefit from medical treatment and/or develop complications. There are many surgical alternatives to surgically treat symptomatic BPH [open prostatectomy, Transurethral resection of the prostate (TUR-P), Transurethral enucleation of the prostate (TUEP), Holmium laser enucleation of the prostate (HoLEP), etc.]. Among these surgical procedures, HoLEP has taken its place as a surgical treatment option of BPH due to its efficacy and safety in large prostates (2). It has been shown that full enucleation performed after HoLEP results in reduced possibility of repeat surgery, less bleeding, and decreased hospital stay due to the shorter duration of catheter use (3). For these reasons, HoLEP surgery has started to be preferred frequently, and its popularity is gradually increasing.

With the current advances in technologies and the widespread use of communication tools, people can now quickly access information on subjects in which they are interested. Video content providers allows patients to easily access information on various treatment methods, which can affect their treatment decisions. YouTube is one of the most popular video-sharing platforms, having more than 1 billion users that collectively watch more than 1 billion hours of videos every day $(4,5)$. It is an ever-developing area where patients receive healthcare information. Unfortunately, information pollution caused by inaccurate information spreading 
through social media tools is a very important issue. Therefore, it is important to ensure the accuracy, reliability and understandability of online information obtained by patients concerning treatment methods. In previous studies, it has already been emphasized that there is a spread of false and/or biased information concerning urological conditions on YouTube $(6,7)$. Video-sharing sites may be suitable for providing information on the surgical treatment of BPH. In particular, videos with animations and informative visual tools can be useful, and current technological developments increasing video quality can help viewers better understand the disease and treatment. However, it can be difficult for patients to distinguish the accuracy of the content of existing from the marketing promises of informing party.

To the best of our knowledge, there is no study in the literature evaluating HoLEP-specific surgical videos on YouTube. Therefore, we aimed to evaluate the content, reliability and quality of the most relevant YouTube videos related to HoLEP surgery.

\section{Materials and Methods:}

\section{Search Strategy and Video Inclusion Criteria}

The keywords 'HoLEP', 'laser enucleation' and 'prostate enucleation' were used to conduct a search on YouTube (http://www.youtube.com) on December 16, 2020 without using any search filter. To reduce bias, all researchers performed the search by clearing their browser's search history and disabling their location status. As the exclusion criteria for the study, videos shorter than 4 minutes, repetitive videos, those with irrelevant content (advertisements, patient references, slide-based presentations, and lectures) and nonEnglish language videos were excluded from the study. A total of 1,416 videos were screened, and 1,156 videos were excluded from the study because they were non-English, irrelevant or non-audio. A further 143 videos were excluded due to duplication. Thus, the number of videos that were eligible was 117 (Figure 1).

\section{Video Parameters and Scoring System}

The videos included in the study were watched by two independent surgeons, both specialized in endourology. In case of inconsistent evaluation between the two surgeons (non-matching results), a third physician evaluated the videos. In addition, the reactions of the viewers to the videos were assessed by recording the parameters of total views, views/month, and video likes and dislikes. The data were divided into two groups based on the source of upload: Group 1 consisted of healthcare providers (doctors, universities, academic journals, university or non-profit physicians, or professional organizations) and Group 2 comprised commercial companies or for-profit organizations. The presence or absence of commercial bias was evaluated as described by Cornish et al. (8). The degree of misinformation was assessed with reference to currently available evidence on surgical BPH treatment as reported by the EAU guidelines (9). In addition, we rated the extent of misinformation of the videos based on a Likert scale of 1 to 5 ('none', 'low', 'moderate', 'high' and 'extreme') (10). All videos were systematically evaluated using the Patient Education Materials Assessment Tool for Audiovisual Materials (PEMAT) and the validated DISCERN quality criteria (11-13).

PEMAT is a systematic method developed to select printable and audiovisual patient education materials, which are easier to understand and easier to act on. We used the version for audiovisual materials, which consists of 13 items measuring understandability and four items measuring actionability. The PEMAT provides two scores for each material - one for understandability and a separate score for actionability. Every item have a 1 point (Agree) or 0 points (Disagree) and N/A was not included the calculation. There is no set cut-off value for the scores.

DISCERN is a standardized index of quality of consumer health information on treatment choices, which can be used by anyone without the need for specialist knowledge. The questionnaire consists of a total of 15 items plus an overall quality rating, with each item representing a separate quality criterion rated from 1 to 5 points (1-2 points: low; 3 points: moderate; and $4-5$ points: high quality). Thus, a total score of 80 points is possible, with higher scores indicating higher quality. For the purposes of this study, we rated the videos using all relevant items and gave them an overall quality rating although not all the videos were directly concerned with treatment choices. 
To determine the overall quality of the videos, the Global Quality Score (GQS), a five-point scale, was used (GQS: $1=$ poor quality; $5=$ excellent quality). This tool measures the accessibility quality and overall flow of the information contained within a video (14).

JAMA is a scoring system with a total of 4 points scored evaluating whether the authors, institutions, references and sources are clearly stated in the video, whether there is information about copyright, whether there is any clear conflict of interest, and whether the dates of uploading and publication are clearly given (15).

\section{Statistical Analysis}

The Statistical Package for the Social Sciences (SPSS) for Windows, version 23.0 was used for the evaluation of the data. The Shapiro-Wilk test was used to determine the normality of distribution. Continuous variables were expressed as median and ranges, and their statistical analysis was performed using the Mann-Whitney $\mathrm{U}$ test. Categorical variables were expressed as number and percentages and analyzed using the chi-square test. Differences were considered statistically significant when the $p$ value was $<0.05$.

\section{Results}

A total of 117 videos were included in the study. The median length of the videos was 14.10 (range = 6.59-30.03) minutes. The median number of views was 590.50 (range $=144.00-2674.00$ ). A significant portion of the videos $(\mathrm{n}=94,77.7 \%)$ had been uploaded by healthcare providers. Although the videos generally focused on surgeons (number of videos $=72,61.5 \%$ ), there were 45 (38.5\%) videos targeting general audience. Table I shows the characteristics of the videos. When the videos were evaluated according to the questionnaires, although the viewing rates differed according to the DISCERN groups, the increase was not linear. Furthermore, the number of views per day and the median number of likes did not differ (Table II). However, when GQS was evaluated, the median values for the number of views, views per day and likes increased in the videos with a GQS of $>3(p=0.019, p=0.019$, and $p=0.009$, respectively).

When the data were divided into Group 1 and Group 2 according to the upload source, the median number of views was 643.50 (range $=155.00-2331.00)$ and $520.00($ range $=181.00-8547.00)$, respectively, indicating a slightly higher value for healthcare providers, albeit with no significant difference $(p=0.916)$ (Table III). There was no statistically significant difference between the upload source groups in terms of the median number of views (per day), median number of likes, reliability score, DISCERN score, and GQS of the videos $(p=0.470, p=0.163, p=0.249, p=0.484$, and $p=0.108$, respectively $)$. However, when PEMAT was evaluated, the understandability scores [Group $1=73.33$ (range $=60.00-92.86$ ) and Group $2=93.33$ (range $=80.00-100.00)]$ and actionability scores [Group $1=75.00($ range $=50.00-100.00)$ and Group $2=100$ (range $=50.00-100.00)]$ were statistically significantly higher in Group $2(p=0.004$ and $p=0.022$, respectively). Furthermore, according to the results of the misinformation scale, Group 1 had uploaded more videos with low-degree misinformation [Group $1=31(34.4 \%)$ ] and Group $2=7(25.9 \%)$ ] while Group 2 was the source of more videos with high-degree misinformation [Group $1=5(5.5 \%)$ and Group $2=9(33.3 \%)]$. There was a statistically significant difference between the two groups in terms of misinformation evaluation $(p=$ $0.001)$.

\section{Discussion}

In this study, we evaluated the quality of YouTube videos on HoLEP that has gained popularity as a frequently preferred surgical method for BPH. Across the world, there are approximately 3 billion internet users that can share information through virtual communication and interaction via the internet in contrast to the more traditional method (16). The huge video archive on YouTube naturally consists of a large number of video contents that examine each subject or topic from different perspectives. However, available evidence has shown that patients can be exposed to low-quality, biased, and/or commercial videos, which can lead to dangerous consequences $(7,17)$ Therefore, it is important to evaluate the reliability and quality of YouTube videos providing health information. Platforms such as YouTube allow patients to easily obtain information about the issues in which they are interested. However, based on the information presented here, 
patients can also make poor decisions or resort to expensive treatments. Nevertheless, the literature shows the increasing viewing of videos about the health field among patients or healthcare professionals (18).

Depending on the upload source of surgical videos on YouTube, the message conveyed to the viewer and its reliability may vary. In a study conducted by Huang et al., it was found that the videos that did not contain accurate information were viewed more and received more comments (19). In another study, it was proven that fake news or inaccurate content spread faster in the internet environment and created more interaction (20). Other researchers similarly emphasized that training videos for skill development might mislead patients due to the presence of unreliable information (21). In our study, regardless of the upload source, we observed that $16.2 \%$ had extreme- or high-degree misinformation while commercial bias was present in $55.7 \%$. In addition, it was observed that complications and alternative treatments were not mentioned in most of the videos. In previous studies, it was shown that the majority of videos uploaded to YouTube were not reliable $(22,23)$. In a review, it was emphasized that most of the health-related YouTube videos presented inaccurate and unreliable information (24). The literature indicates that this misinformation is not necessarily caused by a source being inappropriate or having insufficient expertise, and it could actually be intentional (25). Therefore, we consider that surgical videos on YouTube may pose more of a threat than guidance for patients seeking information to make a treatment decision.

There is no study evaluating the information sources of patients with BPH; therefore, it is not precisely known how the videos posted on video-sharing sites reflect on or affect patients. In previous studies, it was emphasized that the videos uploaded by universities or healthcare institutions provided more comprehensive information and had higher quality $(26,27)$. In a study by Gul et al., the videos were classified as those containing reliable and unreliable information, and the GQS and reliability scores were found to be statistically higher in the former. In addition, the authors showed that the majority of videos containing reliable information had been uploaded by for-profit companies (28). In our study, $23.0 \%$ of the total videos had been uploaded by Group 2. In the literature, it has been reported that the vast majority of educational videos without any financial gain such as those on breast self-examination had been uploaded by universities or physicians, but most on oral leukoplakia had been uploaded by commercial companies for advertisement purposes $(23,29)$. We attribute these differences to the variations of the subjects discussed in videos. In our study, no significant difference was observed in DISCERN, GQS and JAMA scores between the upload source groups. There was also no difference between the two groups in relation to the total number of views, likes and dislikes. A previous study compared videos as useful and misleading, and in contrast to our findings, the authors reported the comprehensiveness score of GQS to be statistically significantly higher in useful videos (21). In the same study, when the data were compared according to the upload source, the GQS, misleading information and comprehensiveness scores were found to be statistically higher for the videos that had been uploaded by for-profit companies (21). In contrast, in our study, we also evaluated the videos using JAMA, PEMAT and Likert scales and found that the PEMAT and misinformation scores were higher in the videos uploaded by Group 2. In a study conducted by Fode et al. to evaluate videos containing medical information, the median PEMAT understandability score was found to be $100 \%$ (range 50-100) and the median PEMAT actionability score was 100\% (range 33-100). It was observed that $28 \%$ of 92 videos containing medical information contained misinformation. The results of their multivariate regression analysis revealed that all the parameters of videos uploaded by medical institutions had a statistically significant effect on DISCERN rating (30). In our study, the PEMAT score differed according to the upload source of the videos. The understandability and actionability scores of the videos uploaded by Group 2 were statistically significantly higher compared to Group 1. Furthermore, although there was misinformation in both groups, the number of videos with high-degree misinformation was significantly higher in Group 2. We consider that the videos uploaded by Group 2 aim to encourage or direct patients to undergo HoLEP surgery, which is a new and expensive treatment. In addition, in the study conducted by Fode et al., it was emphasized that there was no barrier and/or restriction when uploading content to websites, especially in the field of health. In the same study, the authors observed that the majority of the videos had a DISCERN score of 3 or less (30). In a study by Huang et al., there was no difference in the median number of viewers and viewer interaction according to low or high DISCERN scores. However, the authors observed that if a 
video had been uploaded by an academic hospital, it had a higher DISCERN score (19). Similarly, in our study, there was no difference between the DISCERN groups in terms of video viewing parameters. This shows that people watch these videos without distinguishing between poor and good content or they may even not know how to make such a distinction. Thus, the videos they watch can direct them to a wrong treatment or misinformation. The PEMAT score also showed that these videos were easy to understand. Although the easy understandability of a video is a favorable characteristic, misinformation contained in some of the videos can have further negative effects on viewers. The subject of misinformation has been previously investigated and findings similar to our study have been presented by many studies. However, in the literature, the rating of the extent of misinformation as part of video analysis is usually undertaken in a subjective manner, and the rating options are self-designed (e.g., very little, moderate, high and extreme level of misinformation) (25). In our study, all the videos were evaluated independently by two authors. In the evaluation of misinformation, a Likert-type scale was used to minimize possible bias. Inconsistencies were rare, but if any, they were resolved through the evaluation of the third author.

Another cause of concern is the frequent mention of unreliable information and sources of information in viewers' comments related to videos since it can mislead viewers. As emphasized earlier, false information spreads faster on the internet than accurate information. Doctors and healthcare institutions and associations have great responsibility in preventing the spread of such misinformation. Moreover, there are currently no measures to prevent the spread of false information in the comment sections of high-quality videos. Therefore, physicians should direct patients to videos that have been reviewed and proven to be reliable, not only during face-to-face meetings but also through digital interactions, including social media posts, online communications, and telehealth visits. Our findings highlight the importance of high-quality videos that objectively cover all spectrums of a treatment modality and are able to explain it in a way that patients can understand. High-quality information platforms are available (31). In addition, urology associations should be encouraged to upload high-quality and easy-to-understand videos to websites such as YouTube, where patients can research theirs diseases and treatment options.

Videos from a single video-sharing platform (YouTube) were viewed; however, since YouTube is an everevolving website, the evaluation of videos at a single time point may not accurately reflect what patients view after this initial search. By excluding non-English language videos, we may have further reduced the generalizability of our findings. Our study did not include videos available on other online video platforms such as Vimeo or those posted on academic department websites that may not be available on YouTube due to license agreements. Another limitation of the study can be considered as the inability to obtain the demographic characteristics of video viewers. There is still no complete consensus on how to fully evaluate health-related online videos.

\section{Conclusions}

YouTube is one of the popular platforms for presenting healthcare information and developing skills. In light of these results, it is important to evaluate viewers' behavior according to video uploaders. Therefore, the number of videos posted on video-sharing websites such as YouTube presenting accurate and reliable information about diseases and treatments should be increased, and these videos should be allowed to be published after the approval of institutions such as healthcare associations and universities. In addition, YouTube must promptly remove videos that may lead to dangerous treatment for patients or that may contain false information. High-quality educational videos that cover all aspects of treatment options are desperately needed to properly fill the gaps in the YouTube archive. We consider that further studies in urology and other medical fields will contribute to the quality and reliability of health-related video content.

Data availability statement: We can share our data.

Funding statement: There is no funding received for this study.

Conflict of interest disclosure: The authors declared that there is no conflict of interest.

\section{Reference}


1. Bushman W. Etiology, epidemiology, and natural history of benign prostatic hyperplasia. Urol Clin North Am. 2009 Nov;36(4):403-15, v.

2. Elmansy HM, Kotb A, Elhilali MM. Holmium laser enucleation of the prostate: long-term durability of clinical outcomes and complication rates during 10 years of followup. J Urol. 2011 Nov;186(5):1972-6.

3. Michalak J, Tzou D, Funk J. HoLEP: the gold standard for the surgical management of BPH in the 21(st) Century. Am J Clin Exp Urol. 2015;3(1):36-42.

4. YouTube. YouTube in numbers [Webpage] [Internet]. youtube.com. 2020. p. 1-1. Available from: https://www.youtube.com/intl/en-GB/about/press/

5. The top 500 sites on the web. Alexa Internet, Inc; [Internet]. Accessed April 30, 2020. 2020. Available from: https://www.alexa.com/topsites

6. Loeb S, Taylor J, Borin JF, Mihalcea R, Perez-Rosas V, Byrne N, et al. Fake News: Spread of Misinformation about Urological Conditions on Social Media. Eur Urol Focus. 2020 May;6(3):437-9.

7. Loeb S, Sengupta S, Butaney M, Macaluso JNJ, Czarniecki SW, Robbins R, et al. Dissemination of Misinformative and Biased Information about Prostate Cancer on YouTube. Vol. 75, European urology. Switzerland; 2019. p. 564-7.

8. Cornish JK, Leist JC. What constitutes commercial bias compared with the personal opinion of experts? J Contin Educ Health Prof. 2006;26(2):161-7.

9. European Association of Urology. Non-Oncology Guidelines.Managment of Non-neurogenic Male LUTS. In 2020. Available from: https://uroweb.org/guideline/treatment-of-non-neurogenic-male-luts

10. Likert R. A Technique for the Measurement of Attitudes. Arch Psychol. 1932;140:1-55.

11. The patient education materials assessment tool (PEMAT) and user's guide. Rockville: Agency for Healthcare Research and Quality [Internet]. 2020. Available from: https://www.ahrq.gov/healthliteracy/patient-education/pemat-av.html

12. DISCERN Online. Quality criteria for consumer health information; [Internet]. 2020. Available from: www.discern.org.uk/index.php

13. Charnock D, Shepperd S, Needham G, Gann R. DISCERN: an instrument for judging the quality of written consumer health information on treatment choices. J Epidemiol Community Health. 1999 Feb;53(2):10511.

14. Bernard A, Langille M, Hughes S, Rose C, Leddin D, Veldhuyzen van Zanten S. A systematic review of patient inflammatory bowel disease information resources on the World Wide Web. Am J Gastroenterol. 2007 Sep;102(9):2070-7.

15. Silberg WM, Lundberg GD, Musacchio RA. Assessing, controlling, and assuring the quality of medical information on the Internet: Caveant lector et viewor-Let the reader and viewer beware. Vol. 277, JAMA. United States; 1997. p. 1244-5.

16. Petrucci AM, Chand M, Wexner SD. Social Media: Changing the Paradigm for Surgical Education. Clin Colon Rectal Surg. 2017 Sep;30(4):244-51.

17. Sajadi KP, Goldman HB. Social networks lack useful content for incontinence. Urology. 2011 Oct;78(4):764-7.

18. Adhikari J, Sharma P, Arjyal L, Uprety D. YouTube as a Source of Information on Cervical Cancer. N Am J Med Sci. 2016 Apr;8(4):183-6.

19. Huang MM, Winoker JS, Allaf ME, Matlaga BR, Koo K. Evidence-based quality and accuracy of YouTube videos about nephrolithiasis. BJU Int. 2020 Aug; 
20. Vosoughi S, Roy D, Aral S. The spread of true and false news online. Science. 2018 Mar;359(6380):114651.

21. Culha Y, Culha MG, Acaroglu R. Evaluation of YouTube Videos Regarding Clean Intermittent Catheterization Application. Int Neurourol J. 2020 Sep;24(3):286-92.

22. Singh AG, Singh S, Singh PP. YouTube for information on rheumatoid arthritis-a wakeup call? J Rheumatol. 2012 May;39(5):899-903.

23. Esen E, Aslan M, Sonbahar BÇ, Kerimoğlu RS. YouTube English videos as a source of information on breast self-examination. Breast Cancer Res Treat. 2019 Feb;173(3):629-35.

24. Drozd B, Couvillon E, Suarez A. Medical YouTube Videos and Methods of Evaluation: Literature Review. JMIR Med Educ. 2018 Feb;4(1):e3.

25. Betschart P, Pratsinis M, Müllhaupt G, Rechner R, Herrmann TR, Gratzke C, et al. Information on surgical treatment of benign prostatic hyperplasia on YouTube is highly biased and misleading. BJU Int. 2020 Apr;125(4):595-601.

26. Serinken M, Eken C, Erdemir F, Eliçabuk H, Başer A. The reliability of national videos related to the kidney stones on YouTube. Turkish J Urol. 2016 Mar;42(1):7-11.

27. Sood A, Sarangi S, Pandey A, Murugiah K. YouTube as a source of information on kidney stone disease. Urology. 2011 Mar;77(3):558-62.

28. Gul M, Diri MA. YouTube as a Source of Information About Premature Ejaculation Treatment. J Sex Med. 2019 Nov;16(11):1734-40.

29. Kovalski LNS, Cardoso FB, D'Avila OP, Corrêa APB, Martins MAT, Martins MD, et al. Is the YouTube $^{\text {TM }}$ an useful source of information on oral leukoplakia? Oral Dis. 2019 Nov;25(8):1897-905.

30. Fode M, Nolsøe AB, Jacobsen FM, Russo GI, Østergren PB, Jensen CFS, et al. Quality of Information in YouTube Videos on Erectile Dysfunction. Sex Med. 2020 Sep;8(3):408-13.

31. European Association of Urology. Patient information. In. Available from: http://patients.uroweb.org/iam-a-urology-patient/

Table 1. Characteristics of the videos

\begin{tabular}{ll}
\hline & Value \\
Duration (months) $^{\text {a }}$ & $24.00(8.00-53.00)$ \\
Video length (minutes) $^{\text {a }}$ & $14.10(6.59-30.03)$ \\
Total number of views $^{\text {a }}$ & $590.50(144.00-2674.00)$ \\
Number of views per day $^{\text {a }}$ & $0.87(0.18-3.43)$ \\
Number of comments $^{\text {a }}$ & $0(0.00-2.00)$ \\
Number of likes $^{\text {a }}$ & $5.00(1.00-16.00)$ \\
Number of dislikes $^{\text {a }}$ & $0.00(0.00-1.00)$ \\
Misinformation score $^{\text {a }}$ & $3.00(2.00-4.00)$ \\
LIKERT scale $^{\text {a }}$ & $3.00(2.00-4.00)$ \\
GQS score $^{\text {a }}$ & $4.00(3.00-4.00)$ \\
JAMA score $^{\text {a }}$ & $2.00(1.00-2.00)$ \\
PEMAT a $_{\text {Understandability }}$ & $80.00(64.29-93.33)$ \\
Actionability & $75.00(50.00-100.00)$ \\
DISCERN group & \\
Low & $11(9.3 \%)$ \\
Moderate & $29(24.7 \%)$ \\
High & $77(65.7 \%)$
\end{tabular}




\begin{tabular}{|c|c|}
\hline GQS group & $1(0.9 \%)$ \\
\hline GQS 1 & $15(12.8 \%)$ \\
\hline GQS 2 & $28(23.9 \%)$ \\
\hline GQS 3 & $58(49.6 \%)$ \\
\hline GQS 4 & $15(12.8 \%)$ \\
\hline \multicolumn{2}{|l|}{ GQS 5} \\
\hline Misinformation ${ }^{\mathrm{b}}$ & $1(0.8 \%) 14(11.9 \%)$ \\
\hline Severe & $39(33.3 \%)$ \\
\hline High & $38(32.4 \%) 15(12.8 \%)$ \\
\hline \multicolumn{2}{|l|}{ Moderate } \\
\hline \multicolumn{2}{|l|}{ Low } \\
\hline \multicolumn{2}{|l|}{ None } \\
\hline \multirow{4}{*}{\multicolumn{2}{|c|}{$\begin{array}{l}\text { Intended audience }{ }^{\mathrm{D}} \text { Surgeon General } \\
\text { Information presented by }{ }^{\mathrm{b}} \text { Doctor Healthcare } \\
\text { Industry } \\
\text { Other }\end{array}$}} \\
\hline & \\
\hline & \\
\hline & \\
\hline & $99(85.3 \%)$ \\
\hline & $5(4.3 \%)$ \\
\hline & $5(4.3 \%)$ \\
\hline & $8(6.1 \%)$ \\
\hline Discussion of alternative treatment options ${ }^{b}$ & $74(63.2 \%)$ \\
\hline \multicolumn{2}{|l|}{ Absent Exist } \\
\hline \multirow{2}{*}{$\begin{array}{l}\text { Are side effects mentioned in the video? }{ }^{\text {b }} \text { Absent } \\
\text { Exist }\end{array}$} & $64(54.7 \%)$ \\
\hline & $53(45.3 \%)$ \\
\hline \multirow[t]{2}{*}{ Surgical benefits ${ }^{\mathrm{b}}$ Absent Exist } & $5(4.3 \%)$ \\
\hline & $112(95.7 \%)$ \\
\hline Commercial bias ${ }^{b}$ & $51(43.6 \%)$ \\
\hline \multicolumn{2}{|l|}{ Absent } \\
\hline \multicolumn{2}{|l|}{ Exist } \\
\hline \multirow{3}{*}{ Depiction of real surgery ${ }^{b}$ None Exist } & $66(56.4 \%)$ \\
\hline & $9(7.7 \%)$ \\
\hline & $108(92.3 \%)$ \\
\hline
\end{tabular}

a Data expressed as median and range

b Data expressed as number and percentages

GQS: Global quality score, PEMAT: Patient Education Materials Assessment Tool for Printable Material.

Table 2. Video properties by scales

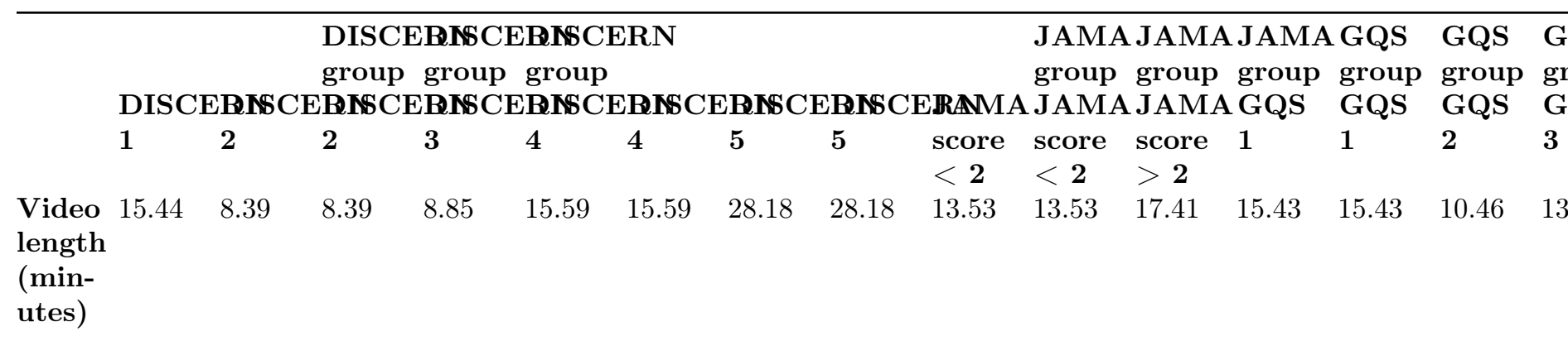




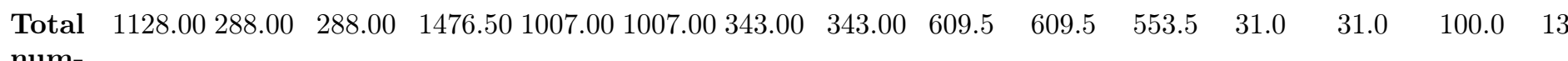
num-

ber

of

views

$\begin{array}{lllllllllllllll}\text { Number.05 } & 0.39 & 0.39 & 0.91 & 1.70 & 1.70 & 1.23 & 1.23 & 0.91 & 0.91 & 0.83 & 0.15 & 0.15 & 0.25 & 1.8 \\ \text { of }\end{array}$

views

per

day

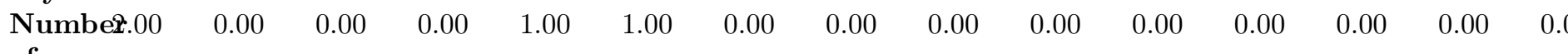

of

com-

ments

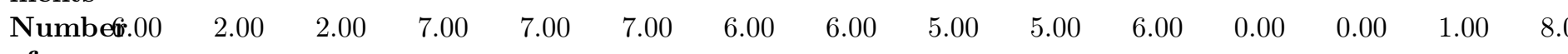

of

likes

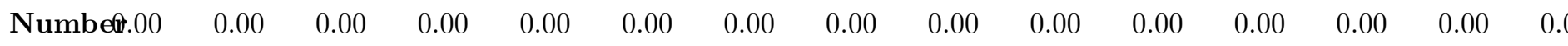

of

dis-

likes

All data expressed as median

Table 3. Comparison of the video data between the upload source groups

\begin{tabular}{|c|c|c|c|}
\hline & $\begin{array}{l}\text { Group } 1 \\
\text { (Healthcare) } \\
(\mathbf{n}=90)\end{array}$ & $\begin{array}{l}\text { Group } 2 \\
\text { (Profit } \\
\text { organizations) } \\
(\mathrm{n}=27)\end{array}$ & $p$ value \\
\hline $\begin{array}{l}\text { Video length } \\
(\text { minutes })^{\mathrm{a}}\end{array}$ & 14. $38(7.22-32.15)$ & $13.22(5.54-20.00)$ & 0.517 \\
\hline $\begin{array}{l}\text { Total number of views } \\
\text { a }\end{array}$ & $643.50(155.00-2331.00)$ & $520.00(181.00-8547.00)$ & 0.916 \\
\hline $\begin{array}{l}\text { Number of views per } \\
\text { day }^{a}\end{array}$ & $1.23(0.33-3.54)$ & $0.76(0.15-2.81)$ & 0.470 \\
\hline Number of comments ${ }^{a}$ & $1.00(0.00-3.00)$ & $0.00(0.00-0.00)$ & 0.038 \\
\hline Number of likes ${ }^{a}$ & $7.00(2.00-18.00)$ & $2.00(0.00-18.00)$ & 0.163 \\
\hline Number of dislikes ${ }^{a}$ & $0.00(0.00-1.00)$ & $0.00(0.00-1.00)$ & 0.891 \\
\hline Reliability score $^{a}$ & $3.38(2.75-3.88)$ & $3.38(2.75-3.75)$ & 0.249 \\
\hline DISCERN score ${ }^{a}$ & $4.0(3.0-4.0)$ & $4.00(3.00-4.00)$ & 0.484 \\
\hline GQS ${ }^{a}$ & $4.0(3.0-4.0)$ & $3.00(3.00-4.00)$ & 0.108 \\
\hline $\mathrm{JAMA}_{\text {score }}{ }^{\mathrm{a}}$ & $2.0(1.0-3.0)$ & $2.00(1.00-2.00)$ & 0.818 \\
\hline JAMA group b & $68(75.5 \%) 22(24.5 \%)$ & $20(74.1 \%) 7(25.9 \%)$ & 0.859 \\
\hline \multicolumn{4}{|l|}{ JAMA score $<2$} \\
\hline \multicolumn{4}{|l|}{ JAMA score $>2$} \\
\hline PEMAT $^{\mathrm{a}}$ & $73.33(60.00-92.86)$ & $93.33(80.00-100.00)$ & 0.004 \\
\hline $\begin{array}{l}\text { Understandability } \\
\text { Actionability }\end{array}$ & $75.00(50.00-100.00)$ & $100(50.00-100.00)$ & 0.022 \\
\hline
\end{tabular}



Misinformation degree ${ }^{b}$
$1(1.1 \%) 5(5.5 \%) 32$
$0(0.0 \%) 9(33.3 \%) 7$
$(35.5 \%) 31(34.4 \%) 11$
$(12.2 \%)$
$(25.9 .0 \%) 7(25.9 \%) 4$
High
Moderate
Low
$(14.8 \%)$
0.001
None

${ }^{a}$ Data expressed as median and range

b Data expressed as number and percentages

GQS: Global quality score, PEMAT: Patient Education Materials Assessment Tool for Printable Materials

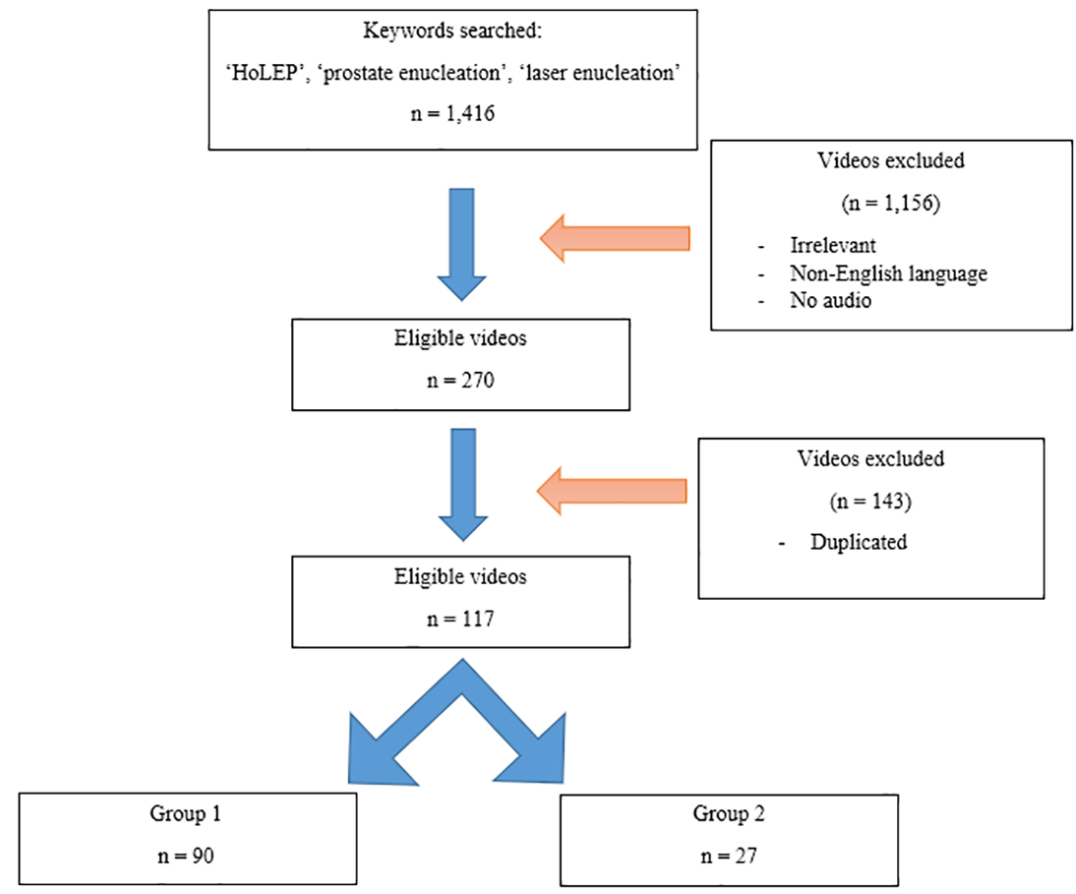

Figure 1. Selection of eligible YouTube videos for the study 